Paris, cNRS Éd., coll. Culture et société, 2010, 590 p.

\title{
Ioana PoPA, Traduire sous contraintes. Littérature et communisme (1947-1989)
}

Miao Chi

\section{(2) OpenEdition}

\section{Journals}

Édition électronique

URL : https://journals.openedition.org/questionsdecommunication/6756

DOI : 10.4000/questionsdecommunication.6756

ISSN : 2259-8901

\section{Éditeur}

Presses universitaires de Lorraine

\section{Édition imprimée}

Date de publication : 1 septembre 2012

Pagination : 322-324

ISBN : 978-2-8143-0120-7

ISSN : 1633-5961

\section{Référence électronique}

Miao Chi, « loana Popa, Traduire sous contraintes. Littérature et communisme (1947-1989) », Questions de communication [En ligne], 21 | 2012, mis en ligne le 18 décembre 2012, consulté le 29 octobre 2022. URL : http://journals.openedition.org/questionsdecommunication/6756 ; DOI : https://doi.org/ 10.4000/questionsdecommunication.6756

\section{(c) $(1)$}

Creative Commons - Attribution - Pas d'Utilisation Commerciale - Pas de Modification 4.0 International - CC BY-NC-ND 4.0

https://creativecommons.org/licenses/by-nc-nd/4.0/ 
gouvernement ont perdu tout crédit « intellectuel » que leur conféraient leurs positions dominantes dans les institutions : l'intellectuel étant d'abord et avant tout, comme le rappelle Gérard Noiriel, celui qui dit la vérité au pouvoir au nom des opprimés. De plus, la critique portée par les intellectuels critiques et les intellectuels spécifiques contribuera à entamer leur audience et leur crédit.

Dans ces conditions, quelle est l'originalité des intellectuels spécifiques? II faut d'abord noter que l'expression « intellectuel spécifique » date des années 70 ; son rôle a été ainsi défini par Michel Foucault, comme le rappelle Gérard Noiriel : « Le travail d'un intellectuel n'est pas de modeler la volonté politique des autres. II est, par les analyses qu'il fait dans les domaines qui sont les siens, de réinterroger les évidences et les postulats, de secouer les habitudes, les manières de faire et de penser » (p. 209). En partant de ce constat avec un regard rétrospectif, on peut dire qu'au fond, le premier intellectuel spécifique avant le concept a été Emile Durkheim. En effet, toute son œuvre peut se lire comme un effort pour échapper au déterminisme de la race très présent à l'époque dans les sciences sociales. C'est pourquoi dans son intervention sur l'affaire Dreyfus, il reste cantonné dans son domaine de compétence sans se prononcer sur l'affaire elle-même. Cette même posture d'intellectuel spécifique se retrouve chez Claude Lévi-Strauss qui a été directeur de l'École (pratique), professeur au Collège de France et membre de l'Académie française : il n'aura de cesse de défendre l'autonomie de la science à l'égard du politique.

Mais les deux intellectuels spécifiques à avoir marqué les dernières décennies du $X X^{e}$ siècle sont Michel Foucault et Pierre Bourdieu. Toutefois, il faut noter que c'est avec Michel Foucault que cette posture de l'intellectuel spécifique se trouve la plus affirmée même si, au fil des analyses, il oscille entre la posture de l'intellectuel critique et celle de l'intellectuel spécifique. Lui aussi est conduit à prôner l'autonomie de la science de sorte que son œuvre apparaît comme une série d'études spécifiques sur des thèmes d'époque (la folie, la prison, la sexualité, etc.) à l'usage des dominés. Chez Pierre Bourdieu, on note un souci d'être utile aux autres qui le conduit à osciller entre une posture d'intellectuel spécifique et une posture d'intellectuel critique pour finalement adopter celle du savant-écrivain désireux de faire partager son expérience telle que proposée par Michel Foucault dans les années 70 : « Et rien ne me rendrait plus heureux que d'avoir réussi à faire que certains de mes lecteurs/lectrices reconnaissent leurs expériences, leurs difficultés, leurs interrogations, leurs souffrances, etc., dans les miennes et qu'ils tirent de cette identification réaliste, ce qui est tout à fait à l'opposé d'une projection exaltée, des moyens de faire et de vivre un tout petit mieux ce qu'lls vivent et ce qu'ils font » (p. 258). Ce retour à soi ou sur soi sonne comme une posture d'authenticité qui, seule, peut parler dans le vrai. II reste qu'aucun de ces intellectuels ne semble pleinement satisfait de son positionnement. On pourrait donc se demander si l'insatisfaction n'est pas ce qui, précisément, définit l'intellectuel (universitaire), voire ce qui l'habite et le hante et, par conséquent, le jette dans une quête inlassable de positionnement, tant la question du savant et du politique nécessite un questionnement continu.

Avec ce livre, Gérard Noiriel offre une plongée dans la vie intellectuelle française depuis l'affaire Dreyfus jusqu'à nos jours. Loin d'être simplement linéaire, l'exposé opère des va-et-vient d'une époque à l'autre ; ce qui permet d'éclairer autrement les enjeux à l'œuvre et les tensions souterraines. Son originalité et sa méthode résident dans la convocation de l'histoire personnelle de chacun des protagonistes. En effet, l'histoire personnelle n'est jamais étrangère à la trajectoire de ces intellectuels. Des éléments biographiques, voire autobiographiques, permettent d'éclairer certaines orientations professionnelles ou options théoriques: des difficultés de socialisation éprouvées dans l'enfance communes aux intellectuels révolutionnaires et aux intellectuels spécifiques aux facteurs sociaux déterminants pour les intellectuels de gouvernement. On note bien dans cet ouvrage de Gérard Noiriel des linéaments pour une sociogenèse des intellectuels français.

Jean Zoungrana

CRESS, université de Strasbourg zoungrana@unistra.fr

loana PoPA, Traduire sous contraintes. Littérature et communisme (1947-1989).

Paris, cnRs Éd., coll. Culture et société, 2010,590 p.

« Rideau de fer 》, « mur 》, « frontière infranchissable » (p. 5), ces métaphores sont liées à la connaissance politique des régimes communistes en Europe de l'Est avant la chute du mur de Berlin. Avec l'application du modèle soviétique aux pays d'Europe de l'Est après la Seconde Guerre mondiale, les conditions de publication et d'exercice du métier d'écrivain ont été transformées par suite du contrôle idéologique et de l'installation d'une censure « préventive et 
répressive » (p. 7). Dans ce contexte rigoureux, qui sont les écrivains traduits à étranger? Par quels circuits ces œuvres passent-elles en traduction et comment celles-ci dépassent des frontières ? Comment les acteurs engagés supportent-ils et surmontent-ils des contraintes politiques, économiques, juridiques, matérielles et linguistiques?

Pour répondre à ces questions, loana Popa, chargée de recherche au CNRS et auteur d'une thèse soutenue à l'École des hautes études en sciences sociales qui est la matière première de cet ouvrage, procède à une étude socio-historique et comparative des transferts littéraires vers la France de 1947 à 1989 en provenance de cinq pays : la Pologne, la Hongrie, la Tchécoslovaquie, la Roumanie et I'Union des républiques socialistes soviétiques (I'URSS). Grâce à l'analyse du processus de traduction, cette étude explore une question originale sur « les modalités de circulation internationale des textes produits dans des contextes de régime autoritaire » (p.7). Situés dans une configuration sociopolitique de production et de réception, dans l'introduction, deux types de circuits de traduction sont distingués : « un espace réglementé » (un écrivain peut publier et circuler ses œuvres nationales et internationales sous contraintes d'un pouvoir politique) et « un espace non autorisé de transfert » (p. 13). Plus précisément, trois souscatégories s'inscrivent dans cet espace réglementé : les circuits d'exportation (publier pour diffuser à étranger), officiel (avoir autorisation officielle pour la circulation internationale) et patrimonial (ayant publié et traduit avant l'instauration des régimes communistes, donc n'ayant pas dû repasser par la censure du nouveau régime). De l'autre côté, selon les différents niveaux de censure, l'espace non autorisé est hiérarchisé par trois genres: les circuits semi-officiel (interdire la traduction d'œuvre publiée à l'étranger), parallèle - traductions faites partir d'un texte publié dans la langue d'origine par samizdat ou des maisons d'édition en exil, soit dans le pays même, soit à l'étranger, « par des instances de diffusion parallèles aux circuits autorisés » (p. 20) - et direct - sans médiation, « la tradition elle-même [...] constitue l'édition originale d'un texte » (p. 2l). Cette classification du circuit de transfert est une méthode essentielle pour faciliter l'analyse du rapport entre la politique culturelle extérieure des pays en Europe de l'Est et la circulation internationale des écrits dans la période concernée.

L'ouvrage est découpé en trois parties qui suivent un ordre chronologique. La première, intitulée « La bipolarisation des enjeux » (p. 25), offre une configuration du transfert littéraire entre 1947 et 1955. À l'intérieur des régimes socialistes, dans le contexte d'une controverse sur le fonctionnement du patrimoine littéraire entrainé par des nouvelles démocraties populaires, l'objectif de « construction d'une image de marque d'un pays à l'étranger dans une espace $[\ldots]$ littéraire international » (p. 33) est privilégié. À l'extérieur, la bipolarisation des relations politiques internationales aggrave la complexité des enjeux de la littérature pratiquée et sa circulation. L'espace du transfert littéraire est donc devenu « un lieu de tension et de concurrence » (p. 28) entre deux blocs, communisme et anticommunisme. Dès lors, les écrivains qui traversent les frontières sont avant tout des membres des délégations des parties communistes ; cependant, pour les auteurs non autorisés ou de l'autre bord politique, les ouvrages de ceux-ci circulent plus difficilement (ibid.). Par rapport au champ d'importation et de réception vers la France, le Parti communiste français (PCF) joue un rôle essentiel dans la configuration de la traduction et du transfert littéraire autorisé avec ses structures éditoriales comme les Éditeurs français réunis (EFR), le Comité national des écrivains (CNE), les revues Les Lettres françaises, L'Humanité, Les Lettres nouvelles, etc. En parallèle, pour échapper au contrôle des régimes autoritaires, pendant la première moitié des années 1950, les médiateurs proches du Congress for Cultura Freedom (CCF) ont mis en place une modalité de contre-engagement : «Traduire en français des livres préalablement publiés dans la langue d'origine de l'autre par des éditeurs en exil » (p. |4I). La partie suivante, « Une ouverture contrôlée » (p. 155), est structurée en quatre chapitres qui abordent la deuxième période de 1956 à 1967 en développant les différentes pistes de cette ouverture contrôlée de transfert : les contours historiques, les effets nationaux et internationaux et les acteurs engagés. Après la mort de Staline, l'intérieur du champ socialiste se manifeste une série de moments critiques et dincertitude sur la politique en URSS, celle qui contribue à une décrispation des relations interculturelles entre Est et Ouest, et surtout à un accroissement de la circulation internationale des ouvrages ; néanmoins, l'intervention soviétique sur les révolutions polonaise et hongroise en 1956 conduit à l'écrasement des mouvements démocratiques. Des pratiques d'écriture et de traduction rencontrent de grandes difficultés sous les contraints politiques de contrôle strict ou d'interdit de l'uRss. Par conséquent, un nouveau circuit de traduction d'Europe de l'Est en Occident a été inventé : la transcription manuscrite (p. 246). Dans cette période, l'écrivain soviétique Boris Pasternak, lauréat du prix Nobel de littérature 
en 1958, est le protagoniste et le témoin qui « ouvre la voie à un mode d'action subversive des écrivains des pays socialistes » (p. 3|0). Traduit d'un manuscrit et publié directement, son roman Le Docteur Jivago (1957) représente une nouvelle modalité de transferts, celle qui provoque une controverse autour de la traduction non-autorisée vers l'Occident et les difficultés d'ordre pratique, politique ou juridique entre des écrivains de l'Est et des éditeurs de l'Ouest de l'Europe (pp. 245-310). Depuis le milieu des années 60, ce schéma est adopté pour des écrivains connus comme Milan Kundera, Pavel Kohout, Paul Goma et Dumitru Tepeneag qui sont tous reçus en Occident en raison de leur dissidence (p. 3/0). Intitulée «Traductions de la contestation » (p. 387), la dernière partie montre la troisième configuration historique (1968-1989). Les crises politiques en 1968 mène au déclin de la puissance des réseaux communistes et leurs appareils partisans dans le transferts littéraire, et donc une restructuration de l'espace du transferts se produit dans des pays d'Europe de l'Est. Cette transformation contribue à « modifier l'équilibre des flux, les choix de traduction, le profil des auteurs traduits et leurs appropriations » (p. 389) dans l'espace intellectuel français. Grâce à l'intensification des aides financières fournies par l'Occident pour soutenir des écrivains dissidents, la traduction et la publication des œuvres non autorisées sont argumentées et renforcées. Cependant, le circuit officiel domine toujours l'importation des traductions en France sous l'influence des effets des politiques culturelles extérieures mises en place par les régimes communistes

Située dans un contexte de domination politique, la recherche d'loana Popa contribue à une exploration approfondie des enjeux politiques sur les transferts culturels et la circulation internationale des ouvrages littéraires et propose une perspective sociohistorique des rapports entre les intellectuels et le pouvoir politique. Elle a également participé à l'essor récent d'une sociologie de traduction avec un certain nombre de chercheurs comme Isabelle Kalinowski (Une Histoire de la réception de Hölderlin en France, thèse de doctorat de Lettres allemandes, université Paris 12, 1999), Johan Heilbron et Gisèle Sapiro (« La traduction comme vecteur des échanges culturels internationaux »», pp. 25-44, in : Sapiro G., dir., 2008, Translatio. Le marché de la traduction en France à l'heure de la mondialisation, Paris, cNRS Éd.), puis Blaise Wilfert (« Cosmpolis et l'homme invisible. Les importateurs de littérature étrangère en France |885-19|4 », Actes de la recherche en sciences sociales, 144, 2002, pp. 33-46)... À travers cette étude et ces travaux antérieurs, est-ce qu'on peut explorer les conditions d'exercice des métiers écrivains dans autres pays socialistes comme la Chine, tester les observations obtenues et interroger sur des rapports entre ces intellectuels et pouvoir politique de leur pays ou encore leur modalité de transfert littéraire vers l'Asie ou l'Europe.

\section{Miao Chi}

CREM, université de Lorraine miao.chi@umail.univ-metz.fr

\section{Médias, journalisme}

Jocelyne Arquembourg, L'événement et les médias. Les récits médiatiques des tsunamis et les débats publics (I 755-2004).

Paris, Éd. des Archives contemporaines, 201 I, 19 | p.

Le livre que Jocelyne Arquembourg a consacré à l'analyse de l'événement à partir du récit médiatique de deux tsunamis catastrophiques mérite d'être lu, questionné,discuté sous un triple point devue.D'abord, il est une réflexion sur l'événement médiatique qui, mettant en son centre la relation entre factualisation et événementialisation, renouvelle fortement cette problématique en s'écartant résolument des perspectives média-centriques, fréquentes lorsqu'il s'agit d'étudier la « construction médiatique » d'une « affaire » ou de « problèmes sociaux ». Ensuite, il place exactement en son centre deux catastrophes, le « tremblement de terre » de Lisbonne en 1755 et les tsunamis de 2004 en Indonésie, ce qui n'est pas une mince affaire, mais permet de questionner à la fois les caractéristiques de l'événement « moderne » et surtout de revenir sur la question du « public » dans une perspective assez éloignée de « l'espace public » habermassien. Enfin, il met en évidence la multiplicité des interactions qui conduisent à une forme de récit médiatique original, notamment du fait que la catastrophe de 2004 intègre une foule d'images d'amateurs qui sont aussi victimes de ce qui se passe alors, et que la prolifération des dons interroge le lien entre l'information et la « solidarité ». C'est dire que ce livre d'une taille raisonnable (191 pages), de lecture assez aisée dans ses premiers chapitres, s'avère de plus en plus dense au fil des pages, et ne saurait se lire d'un trait, tant il mobilise une réflexion extrêmement nourrie. Nommer, catégoriser, déterminer, raconter : ces opérations, on le sait, ne sont guère le décalque d'un réel « en soi ». C'est le point de départ de toute analyse des constructions médiatiques. 